\title{
Polarización de la estructura funcional de las capitales de provincia y de autonomía en España: 1981-2011*
}

\section{Polarization of the functional structure of capital cities of provinces and autonomies in Spain: 1981-2011}

\author{
Severino Escolano Utrilla**
}

\section{INTRODUCCIÓN}

Entre las transformaciones urbanas más importantes de las últimas décadas figuran las registradas en las economías de las ciudades que se han plasmado, tanto en los modos de organización de la producción, la distribución y el consumo, como en la productividad, la composición de los tipos de actividad económica y otros aspectos del «paisaje económico». El estudio de la re-estructuración de las economías urbanas constituye uno de los temas centrales de la investigación científica sobre las ciudades (Moulaert y Scott, 1997, p. 4)

Parte de los cambios de las actividades económicas urbanas pueden ser interpretados considerando las ciudades de un espacio determinado como un sistema de lugares alternativos, en competencia y cooperación, para localizar actividades económicas. En este marco, la composición del mix de actividades económicas de las ciudades, que varía constantemente, obedece a la interacción compleja de múltiples factores y, por tanto, sintetiza bien la posición de una ciudad en la jerarquía urbana y es un indicador del potencial económico-social, del grado de modernización y otras propiedades de las economías urbanas.

*Este texto se enmarca en el proyecto de investigación «Las ciudades españolas en la etapa autonómica (1978-2008). Dinámicas, procesos y políticas». CSO 2009-11261-(Subprograma GEOG), del Ministerio de Ciencia e Innovación.

${ }^{* *}$ Universidad de Zaragoza. 
Por otro lado, desde hace dos décadas la innovación tecnológica y el conocimiento científico se han revelado como fuerzas emergentes y decisivas tanto para mejorar la competitividad de ciudades y territorios a todas las escalas como para impulsar la modernización de la sociedad en general.

Alguna de las tendencias fundamentales de la reorganización reciente de las actividades económicas en España se definen por la creciente concentración en determinadas áreas urbanas de ciertas actividades económicas, en especial las relacionadas con la creación, gestión y uso de la innovación, el conocimiento y la información, y por la descentralización, también selectiva, de otras.

En este trabajo se estudia el alcance de estas tesis referidas a los municipios capitales de provincia y de autonomía entre 1981 y 2011. En concreto se pretende responder a las tres preguntas siguientes:

1. ¿Cuál ha sido la variación de los perfiles de actividad económica de las capitales de provincia y de autonomía entre 1981 y 2011 ?

2. ¿Se pueden identificar tipos de trayectorias seguidas por las ciudades en la conformación de sus perfiles de actividad económica?

3. ¿Se aprecian patrones nítidos de cambio y de especialización asociados a la función de capitalidad autonómica?

Los contenidos de este trabajo están organizados en tres partes, además de la introducción y las consideraciones finales. En la primera se presentan algunos procesos y factores que configuran la mezcla de actividades económicas urbanas y se fundamenta la delimitación espacial y temática así como el enfoque de estudio adoptado. La siguiente se dedica a describir y justificar la metodología utilizada y a caracterizar los datos. En la tercera se exponen y explican los resultados de los análisis. Finalmente se realizan algunas valoraciones acerca del grado y modo de influencia de la capitalidad autonómica en la especialización funcional de las ciudades.

\section{FACTORES Y TENDENCIAS DE LA ESTRUCTURA FUNCIONAL Y ESPACIAL DE LAS ACTIVIDADES ECONÓMICAS DE LAS CIUDADES}

La composición de los tipos de actividad económica de las ciudades en un momento dado es el resultado de un balance instantáneo entre varias categorías relativamente simples: la creación de nuevas empresas, la desaparición de otras y el saldo de traslados hacia y desde otras ciudades, a lo que cabría añadir los cambios de actividad. Sin embargo, los movimientos de estas variables es- 
tán sujetos a las complejas interacciones de diversas fuerzas que cristalizan en modelos espaciales específicos de localización de las actividades económicas.

La estructura y variación de los perfiles de actividad económica de las ciudades se explica por la actuación conjunta de factores que, tradicionalmente, se han considerado que conforman los procesos de localización de las actividades económicas: las ventajas competitivas de las economías de aglomeración y «desaglomeración», la accesibilidad a los mercados de factores de producción, las configuraciones de la redes urbanas a diversas escalas, la posición y distancia física y funcional entre ciudades, el estado y dotación de infraestructuras y, en fin, las políticas económicas y de ordenación urbana y territorial, entre otras.

Algunos enfoques de la teoría económica han destacado la importancia de la estructura económica en el desarrollo socioeconómico de los países, regiones y ciudades, es decir, asocian el potencial socioeconómico de un área o una ciudad al mayor o menor peso relativo que las diversas actividades tienen en la composición de la estructura económica de cada entidad.

Diversos factores modifican constantemente la importancia absoluta y relativa de los principios de localización espacial y, en consecuencia, las ventajas de localización de las ciudades. Ello hace que los patrones de localización incrementen su complejidad debido a la combinación de gradientes de actividad asociados a la contigüidad con discontinuidades espaciales generadas por la alta conectividad digital y de los modernos sistemas de transportes y comunicaciones.

\subsection{La influencia de la integración socioeconómica y del conocimiento científico}

La localización de las actividades económicas en las ciudades está influida por múltiples procesos interrelacionados, entre los que sobresalen la creciente internacionalización de la economía y la sociedad y la emergencia de nuevas fuerzas económicas y sociales relacionadas con las innovaciones tecnológicas y el conocimiento científico. Además, otras dimensiones urbanas, de naturaleza económica, demográfica, paisajística y espacial están afectadas también por la intervención de estos dos vectores.

La inserción de las economías urbanas en mercados globales promueve la aparición de nuevas conexiones de todo tipo, de alcance planetario, continental, nacional, regional y local, establecidas entre una multitud de agentes (países, bloques de países, organizaciones, empresas, personas), lo que facilita 
nuevas divisiones del trabajo y especializaciones económicas que operan en nuevos escenarios de competitividad y cooperación. La globalización significa también la aceleración de los procesos económicos, la integración de los sistemas financieros, la reorganización funcional y espacial de los procesos de producción facilitadas por las innovaciones tecnológicas y, en fin, la creciente importancia de actividades económicas relacionadas con la creatividad, el conocimiento y la tecnología (Drucker, 1969; Stehr, 1994; Jonhston, Taylor et al., 2002; UNESCO, 2005).

La integración de España en la Unión Europea (UE) constituye un hecho relevante que modifica las condiciones y ventajas de localización de actividades económicas en buena parte de las grandes ciudades españolas. La ampliación del espacio económico, político y administrativo facilita la reorganización de las redes urbanas y con ello procesos de centralización y descentralización de actividades a escala de la UE.

El proceso de restructuración político administrativa que supone la construcción del «Estado de las Autonomías», iniciado en 1978, afecta específicamente a las ciudades españolas. La transferencia de personas, medios y funciones desde el Estado central a las instituciones autonómicas para el ejercicio de sus competencias acarrea también un incremento de la actividad económica, principalmente en las capitales de autonomía. No obstante, y como se indicó en otro trabajo (Escolano, 2012) es difícil identificar y cuantificar los efectos económicos y de otro tipo producidos por la capitalidad autonómica más allá de la contabilización de los medios materiales y personales asociados a la misma, pues este hecho crea externalidades de diversa naturaleza y muy ramificadas por el tejido socioeconómico. En todo caso, se puede aceptar que la capitalidad de autonomía genera nuevas oportunidades para el desarrollo de actividades económicas.

La creación y aplicación del conocimiento científico y de innovaciones tecnológicas, configuran otro potente conjunto de fuerzas que influyen en la estructura funcional y espacial de las actividades económicas urbanas de múltiples modos: inciden, de forma indirecta, a través de movimientos de cambio que impulsan en otras muchas actividades y, de modo directo, mediante la formación de nuevos subsectores de actividad muy dinámicos.

En efecto, se ha constatado la influencia significativa de estas actividades en el crecimiento económico y en la mejora de la competitividad a todas las escalas (Korres, 2009; Varga, 2009; Cooke, Asheim et al., 2011), su considerable impacto en la organización social y del trabajo (Coats, 2005; Coats y Lekhi, 2008), así como la importancia de la política institucional en el nacimiento y consolidación de este tipo de actividades. También es destacable la 
desigual incidencia social (brecha digital: digital divide) y espacial de las actividades de la economía del conocimiento (Hepworth, 1986; EUROSTAT, 2011; Korres, Tsobanoglou et al., 2011).

Por otra parte, las innovaciones tecnológicas y de organización de los sistemas de producción, distribución y consumo han promovido el nacimiento de nuevas actividades (investigación científica, conocimiento, actividades creativas) o han renovado los modos de producción de otras tradicionales (financieras, seguros), de tal forma que se ha generado un único proceso productivo bienes-servicios, integrado y continuo (Barcet, Bonamy et al., 1984; Bailly y Coffey, 1994). Esta mayor fluidez hace que se difumine la tradicional especialización sectorial, que se desplaza hacia la especialización funcional definida por agrupamientos espaciales de funciones económicas determinadas (Duranton y Puga, 2002; Halbert, 2005).

La difusión de las aplicaciones del conocimiento y de la innovación tecnológica ha impulsado cambios notables en los patrones de localización de muchas actividades económicas (Mandeville, 1983; Castells, 1995; Yigitcanlar, 2009). Las ciudades, por su considerable tamaño demográfico y económico, por su gran diversidad socioeconómica y su buena conectividad, son los lugares más adecuados para la localización de las actividades de la economía del conocimiento y para el desarrollo pleno del potencial de transformación social, económico y espacial que éstas llevan incorporado (Mitchell, 1995; Moss, 1998).

La internacionalización de la economía y los avances tecnológicos confluyen también en la consolidación de otra característica fundamental de las economías urbanas como es el creciente peso económico de los servicios y su omnipresencia en la vida de las ciudades. Su pujanza se debe, entre otras causas, al incremento de la demanda de servicios por los individuos, las familias y las empresas, a la externalización de servicios que antes formaban parte de la actividad industrial y agraria y al crecimiento de los servicios a la colectividad (educativos, sanitarios, culturales, asistenciales) prestados por las instituciones o por empresas. El grupo de los «servicios a las empresas» ha alcanzado también un notable desarrollo; por otro lado, los «servicios avanzados a la producción» (investigación, seguros, auditoría, asesoría jurídica, etc.) y los servicios financieros han adquirido una gran importancia estratégica, dada su contribución esencial a la mejora de la competitividad de las empresas.

Las considerables implicaciones de los procesos señalados en las economías urbanas avalan el empleo de la «intensidad de conocimiento y el tipo de tecnología» utilizada en la producción de bienes y servicios como criterios principales de la clasificación de actividades, combinados con la desagrega- 
ción de los servicios en varias categorías según su orientación al mercado final o a las empresas. Este enfoque permite la inclusión en los análisis de gran variedad de actividades económicas que representan la diversidad de la estructura de las economías urbanas de forma bastante satisfactoria; la magnitud se ha medido mediante la población ocupada en cada rama de actividad.

\subsection{Algunas tendencias de la localización de las actividades económicas en las ciudades}

El desarrollo conjunto de las tendencias apuntadas y otras han creado nuevas lógicas de descentralización y centralización así como requisitos de localización de las actividades económicas que, articulados con ciertas determinaciones de políticas nacionales, regionales y locales, han modificado la especialización económica de las ciudades y regiones.

En general, los perfiles de especialización económica de las ciudades forman parte de los patrones complejos de localización de actividades caracterizados, grosso modo, por la concentración espacial de ciertas actividades en grandes ciudades y por la dispersión selectiva de otras por ciudades medianas y espacios rurales.

En consecuencia, determinadas actividades tienden a concentrarse en grandes áreas urbanas que ocupan los puestos de cabeza de las jerarquías urbanas nacionales e internacionales, en tanto que otras se dispersan por ciudades más pequeñas, generalmente bien conectadas a las anteriores por modernos sistemas de transportes y comunicaciones.

Abundantes investigaciones empíricas han mostrado la tendencia a la concentración en grandes ciudades de diversas actividades creativas y de la economía del conocimiento (May, 1997, p. 63; Scott, 1997; Acs, 2006; Glazer y Grimes, 2009), pero también han constatado su difusión selectiva por ciudades medias e incluso por espacios rurales (Méndez, Sánchez et al., 2009; Clayton y Morris, 2010; Puissant y Lacour, 2011; Méndez, Michelini et al., 2012).

En el mismo sentido, el crecimiento y diferenciación de actividades de servicios, los avances en las comunicaciones digitales y otros factores han promovido, simultáneamente, la revalorización de los centros urbanos como asiento de sedes de empresas, centros de decisión, oficinas, etc., y de áreas periféricas bien comunicadas para la instalación de centros comerciales, oficinas administrativas, centros logísticos, conglomerados de industrias y servicios de alta tecnología y otras. Estas tendencias se plasman con desigual intensidad en 
el espacio urbano según el tamaño (demográfico, económico, espacial) de las ciudades y su posición en las redes urbanas: son muy nítidas en las grandes entidades urbanas (áreas metropolitanas, ciudades, región...) y se difuminan en las ciudades medianas y pequeñas.

En resumen: los centros de las ciudades reúnen ventajas de localización para algunas actividades, como servicios avanzados a la producción, servicios financieros y actividades creativas y de la economía del conocimiento, mientras que diversos sectores de las periferias urbanas son más competitivos para la localización de actividades comerciales, de oficinas para desarrollar tareas administrativas, de diferentes tipos de industria, servicios de alta tecnología y centros logísticos.

Esta organización espacial de las actividades económicas tiene consecuencias metodológicas en la delimitación de las unidades de análisis. El uso de los municipios, como en el presente estudio, sólo recoge la dinámica de las áreas centrales en el caso de las grandes áreas metropolitanas y no la totalidad de los procesos espaciales y funcionales.

A otra escala, la configuración de los perfiles de las actividades económicas de las ciudades está estrechamente asociada a los movimientos que se producen en la jerarquía urbana. La articulación de la jerarquía urbana de las ciudades españolas está sujeta, por una parte, a la intervención de fuerzas de integración a escala global, europea, nacional y regional, que afectan con intensidad desigual a cada ciudad. Por otra, diversas actuaciones de variado alcance impulsan también otros procesos de integración. Entre las principales cabe destacar la mejora de las infraestructuras y redes de trasportes y comunicaciones, en espacial el desarrollo de la red ferroviaria de alta velocidad (AVE) (Ureña, 2012). La reorganización territorial de la administración pública y del poder político (Moreno y Escolano, 1992, pp. 91-141) llevada a cabo con la creación del estado de las autonomías ha favorecido la integración regional. La constitución de redes de ciudades interconectadas, de diversos niveles jerárquicos y alcance funcional y espacial variable, ha generado ventajas y externalidades asociadas a la contigüidad y a la conectividad que conforman nuevos y cambiantes patrones espaciales de oportunidades para la localización de actividades económicas.

Para aprehender esta cualidad dinámica de las estructuras de las actividades económicas de las ciudades, el presente trabajo se centra en el estudio de las trayectorias temporales e indaga, en la medida que los datos lo permiten, en los cambios recientes de la composición de las actividades económicas de las capitales de provincia y de autonomía y sus itinerarios de diferenciación que han dado lugar a la configuración actual de los perfiles de actividad. 
Los tipos de actividad se consideran como un datum, resultado de decisiones de localización. La investigación, de carácter eminentemente exploratorio y empírico, está guiada por tres hipótesis fundamentales que caracterizan las tendencias espaciales de los recientes procesos de restructuración y globalización socioeconómicos. La primera se refiere a la creciente concentración económica, política y cultural en las ciudades (metropolitanización) (Veltz, 1997, p. 82; Krätke, 2007), en especial de las actividades relacionadas con el conocimiento, la creatividad, la investigación y la innovación tecnológica. La segunda, conectada con la anterior, denota las trayectorias diferenciadas que han seguido las capitales provinciales y autonómicas en la conformación de sus perfiles de especialización funcional: la capitalidad de autonomía figura entre los factores que intervienen en la especialización económica. La tercera concierne a la escala de los procesos: la conformación de los perfiles de actividad de algunas ciudades no puede explicarse completamente si sólo se enmarcan a escala nacional o subnacional; ciertas actividades encuentran ventajas de localización en ciudades que forman parte de redes urbanas de alcance europeo o mundial.

\section{Metodología y datos}

Un estudio de carácter empírico con datos geográficos como el presente requiere que se justifiquen la delimitación de las unidades espaciales, los indicadores del objeto de estudio y los procedimientos analíticos utilizados.

La unidad de análisis empleada en este trabajo es el municipio. Su elección se basa en la existencia de series de datos diacrónicos y sincrónicos consistentes. También es un individuo adecuado para reflejar el alcance de las políticas locales. Por otra parte, son bastantes los estudios de localización de actividades económicas que utilizan el municipio como unidad de análisis, pues permite comparar resultados producidos por diferentes enfoques (Méndez, Sánchez et al., 2009). Sin embargo, es necesario indicar que esta delimitación administrativa puede cercenar algunas conexiones en aquellas áreas urbanas compuestas por varios municipios que funcionan de forma unitaria, y por tanto el municipio solo refleja una imagen parcial del fenómeno estudiado.

La elección de los municipios capitales de provincia y de autonomía y las dos ciudades autónomas es congruente con los objetivos del trabajo, que buscan identificar y delimitar la posible influencia de la capitalidad autonómica en la estructura de sus perfiles de actividad económica. Esta muestra de ciuda- 
des, que tienen en común su función administrativa, constituye un conjunto adecuado para la investigación.

El tipo y magnitud de actividad económica se han medido por la población ocupada en ramas de actividad desglosadas a dos dígitos de la Clasificación Nacional de Actividades Económicas (CNAE-93, rev. 1) (INE, s/f). Se ha considerado que este nivel de detalle es suficiente para alcanzar los objetivos propuestos. Los datos de 2011 corresponden a los afiliados a todos los regímenes de la Seguridad Social.

Para formar los perfiles funcionales de las capitales provinciales y autonómicas se han ignorado algunas actividades y las restantes se han agrupado según los criterios siguientes: intensidad de conocimiento, de investigación; grado de innovación tecnológica aplicados en la actividad; orientación de la producción al mercado final o a las empresas (Tabla 1). Esta clasificación es la utilizada por EUROSTAT (EUROSTAT, s/f) y por Krätke (Krätke, 2007) con ligeras modificaciones.

La interpretación de los resultados tiene en cuenta la evolución tecnológica habida en los últimos 30 años que abarcan los datos utilizados, pues algunas actividades, clasificadas en la misma rama en 1981 y 2011, tienen hoy modos de organización y sistemas técnicos bien diferentes de los del año base de comparación (por ejemplo, las telecomunicaciones).

La agrupación realizada reduce la gran variedad de ramas de actividad especificadas con dos dígitos y permite también acomodar los cambios habidos en las clasificaciones. Los datos del censo de 2001 se corresponden con la agrupación expresada en la tabla 1. Los datos de 1981 y 2011 han podido ser asignados a los grupos establecidos con errores menores que, en todo caso, se difuminan cuando se calculan los coeficientes de localización. En cambio, no ha sido posible encajar los datos de 1991 en los grupos fijados, pues la clasificación a dos dígitos comprende 29 ramas de actividad.

Para comparar la intensidad de la especialización de las actividades de cada ciudad en relación con el conjunto de ciudades que sirve de marco de referencia, se ha utilizado el «coeficiente de localización»(QL), cuya razón matemática se expresa:

$$
Q L_{i j}=\frac{X_{i j} / \Sigma_{i=1}^{\mathrm{n}} X_{i j}}{\sum_{j=1}^{\mathrm{m}} X_{i j} / \Sigma_{j} \Sigma_{j} X_{i j}}
$$

Dónde: $\mathrm{QL}_{\mathrm{ij}}$ : Coeficiente de localización de la actividad $j$ en la ciudad $i ; \mathrm{X}_{\mathrm{ij}}$ : Ocupados en la actividad $i$ en la ciudad $j ; \Sigma_{\mathrm{i}} \mathrm{X}_{\mathrm{ij}}$ : Ocupados en todas las activi- 
TABLA 1

GRUPOS DE ACTIVIDAD Y RAMAS QUE COMPRENDEN. DATOS DE 2001

\begin{tabular}{|c|c|}
\hline Grupos & Rama de actividad 2 dígitos CNAE-93 rev 1.1. (2001) \\
\hline \multicolumn{2}{|c|}{ 1. Industrias manufactureras intensivas en investigación } \\
\hline $\begin{array}{l}\text { 1.1. Industrias con uso intensivo } \\
\text { de investigación y tecnología avan- } \\
\text { zada }\end{array}$ & $\begin{array}{l}\text { 30: Fabricación de máquinas de oficina y equipos in- } \\
\text { formáticos; 32: Fabricación. de material electrónico; } \\
\text { fabricación de equipo y aparatos de radio, televisión y } \\
\text { comunicaciones; 33: Fabricación de equipo e instru- } \\
\text { mentos médico-quirúrgicos, de precisión, óptica y } \\
\text { relojería }\end{array}$ \\
\hline $\begin{array}{l}\text { 1.2. Industrias con uso intensivo } \\
\text { de investigación y tecnología de } \\
\text { nivel medio alto }\end{array}$ & $\begin{array}{l}\text { 24: Industria química 29: Industria de la construcción } \\
\text { de maquinaria y equipo mecánico; 31: Fabricación de } \\
\text { maquinaria y material eléctrico; 34: Fabricación de } \\
\text { vehículos de motor, remolques y semirremolques; 35: } \\
\text { Fabricación de otro material de transporte. }\end{array}$ \\
\hline \multicolumn{2}{|c|}{ 2. Industrias con baja intensidad en investigación } \\
\hline $\begin{array}{l}\text { 2.1. Industrias con intensidad baja } \\
\text { en investigación y tecnología de } \\
\text { nivel medio bajo }\end{array}$ & $\begin{array}{l}\text { 15: Industria de productos alimenticios y bebidas 16: } \\
\text { Industria del tabaco; 17: Industria textil; 18: Industria } \\
\text { de la confección y de la peletería; 19: Preparación cur- } \\
\text { tido y acabado del cuero; fabricación de artículos de } \\
\text { marroquinería y viaje; artículos de guarnicionería ta- } \\
\text { labartería y zapatería; 20: Industria de la madera y del } \\
\text { corcho, excepto muebles; cestería y espartería; } 21 \text { : In- } \\
\text { dustria del papel; 22: Edición, artes gráficas y repro- } \\
\text { ducción de soportes grabados; 23: Coquerías, refino } \\
\text { de petróleo y tratamiento de combustibles nucleares; } \\
\text { 25: Fabricación de productos de caucho y materias } \\
\text { plásticas; 26: Fabricación de otros productos mi- } \\
\text { nerales no metálicos; } 27 \text { : Metalurgia; 28: Fabricación } \\
\text { de productos metálicos, excepto maquinaria y equipo; } \\
\text { 36: Fabricación de muebles; otras industrias manufac- } \\
\text { tureras; } 37 \text { : Reciclaje. }\end{array}$ \\
\hline
\end{tabular}

Fuente: Eurostat (S/F) y Krätke (2007).

Estudios Geográficos, Vol. LXXV, 276, pp. 139-175, enero-junio 2014

ISSN: 0014-1496, eISSN: 1988-8546, doi: 10.3989/estgeogr.201404 


\section{TABLA 1 (continuación)}

GRUPOS DE ACTIVIDAD Y RAMAS QUE COMPRENDEN. DATOS DE 2001

\begin{tabular}{|c|c|}
\hline Grupos & Rama de actividad 2 dígitos CNAE-93 rev 1.1. (2001) \\
\hline \multicolumn{2}{|c|}{ 3. Servicios con uso intensivo de conocimiento } \\
\hline $\begin{array}{l}\text { 3.1. Servicios con uso intensivo de } \\
\text { conocimiento y tecnología avanzada }\end{array}$ & $\begin{array}{l}\text { 64: Correos y telecomunicaciones; 72: Actividades in- } \\
\text { formáticas; 73: Investigación y desarrollo. }\end{array}$ \\
\hline $\begin{array}{l}\text { 3.2. Servicios con uso intensivo de } \\
\text { conocimiento orientados al mer- } \\
\text { cado y las empresas }\end{array}$ & $\begin{array}{l}\text { 61: Transporte marítimo, de cabotaje y por vías de } \\
\text { navegación interiores; 62: Transporte aéreo y espacial; } \\
\text { 70: Actividades inmobiliarias; 71: Alquiler de } \\
\text { maquinaria y equipo sin operario, de efectos perso- } \\
\text { nales y enseres domésticos; } 74 \text { : Otras actividades em- } \\
\text { presariales. }\end{array}$ \\
\hline 3.3. Servicios financieros & $\begin{array}{l}\text { 65: Intermediación financiera, excepto seguros y } \\
\text { planes de pensiones; 66: Seguros y planes de pen- } \\
\text { siones, excepto seguridad social obligatoria; 67: Ac- } \\
\text { tividades auxiliares a la intermediación financiera. }\end{array}$ \\
\hline $\begin{array}{l}\text { 3.4. Servicios de salud, educación, } \\
\text { sociales y culturales }\end{array}$ & $\begin{array}{l}\text { 80: Educación 85: Actividades sanitarias y veterina- } \\
\text { rias, servicio social; 92: Actividades recreativas, cul- } \\
\text { turales y deportivas. }\end{array}$ \\
\hline \multicolumn{2}{|c|}{ 4. Servicios con baja intensidad en uso del conocimiento } \\
\hline $\begin{array}{l}\text { 4.1. Servicios con intensidad baja } \\
\text { en uso del conocimiento }\end{array}$ & $\begin{array}{l}\text { 50: Venta, mantenimiento y reparación de vehículos de } \\
\text { motor, motocicletas y ciclomotores; venta al por menor } \\
\text { de combustible para vehículos de motor; } 51 \text { : Comercio } \\
\text { al por mayor e intermediarios del comercio, excepto de } \\
\text { vehículos de motor y motocicletas; } 52 \text { : Comercio al por } \\
\text { menor, excepto el comercio de vehículos de motor, } \\
\text { motocicletas y ciclomotores; reparación de efectos per- } \\
\text { sonales y enseres domésticos; } 55 \text { : Hostelería; 60: Trans- } \\
\text { porte terrestre; transporte por tuberías; } 63 \text { : Actividades } \\
\text { anexas a los transportes; actividades de agencias de via- } \\
\text { jes; } 75 \text { : Administración pública, defensa y seguridad } \\
\text { social obligatoria; 90: Actividades de saneamiento } \\
\text { público; } 91 \text { : Actividades asociativas; } 93 \text { : Actividades di- } \\
\text { versas de servicios personales; 95: Actividades de los } \\
\text { hogares como empleadores de personal doméstico; } 99 \text { : } \\
\text { Organismos extraterritoriales. }\end{array}$ \\
\hline
\end{tabular}


dades de la ciudad $j ; \Sigma_{\mathrm{j}} \mathrm{X}_{\mathrm{ij}}$ : Ocupados de la actividad $i$ en todas las ciudades; $\Sigma_{\mathrm{i}}$ $\Sigma_{\mathrm{j}} \mathrm{X}_{\mathrm{ij}}$ : Ocupados todas las actividades en todas las ciudades.

El valor de coeficiente de localización es siempre positivo: cuando supera la unidad en un tipo de actividad y en un lugar se puede considerar que existe cierto grado de especialización en tal actividad y lugar. Los perfiles funcionales se han formado con los valores del coeficiente de localización de cada grupo de actividad. Se ha considerado que un grupo presenta cierto grado de especialización cuando el coeficiente de localización es igual o superior a 1,1.

\section{PRocesos de CONVERGENCIA Y DIFERENCIACión DE LOS PERFILES DE ACTIVIDAD DE LAS CAPITALES DE AUTONOMÍA Y DE PROVINCIA}

Las cifras de la distribución de la población y los ocupados y afiliados a la Seguridad Social permiten constatar la considerable magnitud de las capitales de autonomía y de provincia en términos absolutos, y cierto grado de concentración de la actividad económica en las mismas. En 2011, la cuarta parte de los afiliados a la Seguridad Social en España residían en las capitales de autonomía, frente a la quinta parte de la población total (Tabla 2).

La evolución registrada entre 1981 y 2011 muestra la continuada y modesta pérdida de peso demográfico y de ocupación de los municipios capitales de autonomía y su mantenimiento en los que son capitales de provincia. Estas variaciones han de interpretarse como parte de un proceso general y complejo de descentralización de la población (Reques y Rodríguez, 1998, pp. 63-77; Instituto Geográfico Nacional, 2008, pp. 236-279; Reques, 2011) y de la actividad económica (Méndez y Pascual, 2006).

La descentralización de la actividad ha ido acompañada de cambios en la composición por grupos de actividad de los ocupados en los 20 años que median entre 1981 y 2001 y de los afiliados a la Seguridad Social. Los datos de la tabla 3 revelan los principales movimientos espaciales y de especialización funcional que diferencian a las capitales de autonomía y provincia entre sí y del resto de los asentamientos.

Entre los rasgos más importantes de la estructura de la ocupación por grupos de actividades cabe destacar el descenso, en valores absolutos y relativos, de los ocupados en actividades industriales con «uso intensivo de investigación y tecnología avanzada y uso intensivo de investigación y tecnología de nivel medio» (grupos 1.1. y 1.2.) y el crecimiento generalizado de los servicios, en especial los que emplean de forma intensiva conocimiento y tecnología avanzada (grupo 3.1.) y los servicios a las empresas (grupo 3.2.). 


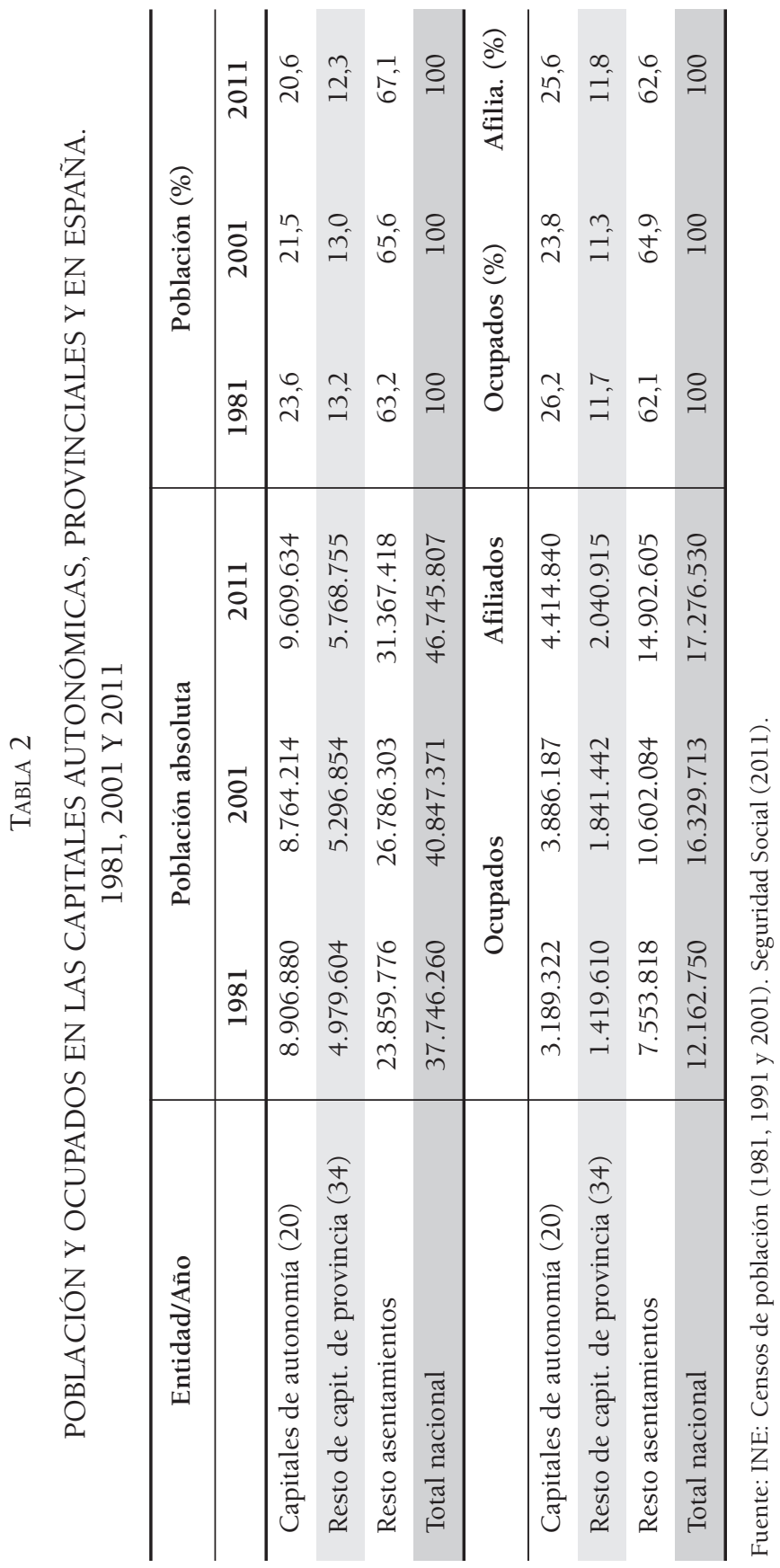

Estudios Geográficos, Vol. LXXV, 276, pp. 139-175, enero-junio 2014 ISSN: 0014-1496, eISSN: 1988-8546, doi: 10.3989/estgeogr.201404 


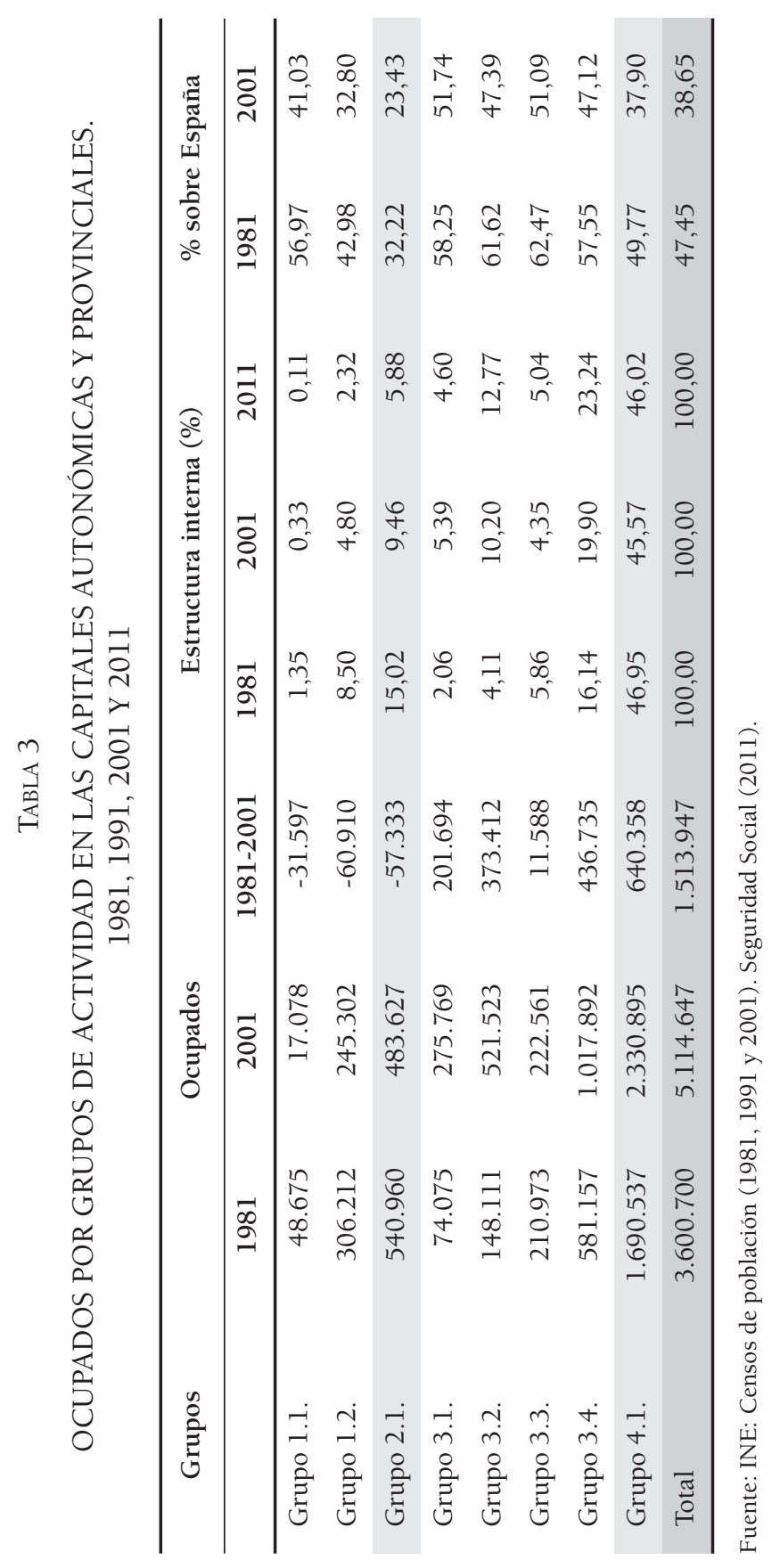

Estudios Geográficos, Vol. LXXV, 276, pp. 139-175, enero-junio 2014

ISSN: 0014-1496, eISSN: 1988-8546, doi: 10.3989/estgeogr.201404 
La contracción de la ocupación en los grupos industriales mencionados y la expansión de los servicios a las empresas se deben, en parte, a la externalización de funciones en empresas industriales. Además hay que sumar el incremento de la demanda de servicios tecnológicos por las empresas y particulares, y el crecimiento de los servicios colectivos a la población (sanitarios, educativos, culturales, sociales).

Otra característica relevante de la variación de la estructura ocupacional por grupos de actividad se encuentra en la menor proporción que tienen en las capitales de autonomía y de provincia las industrias con uso intensivo de conocimiento y de tecnología avanzada y de nivel medio (grupos 1.2. y 2.1.) en comparación con al resto de los asentamientos, frente al mayor peso que alcanzan en las capitales los servicios con uso intensivo de conocimiento y tecnología avanzada y los orientados a las empresas (grupos 3.1. y 3.2.).

Esta aproximación general permite afirmar que los municipios capitales de autonomía y de provincia son localizaciones muy atractivas para empresas de servicios relacionados con la creación y uso del conocimiento y la tecnología y por tanto, son centros impulsores de innovación y de actividad económica. También muestra la salida de actividades industriales a otras ciudades de la periferia de las grandes áreas urbanas y de ciudades intermedias.

\subsection{Crecimiento desigual de los grupos de actividad económica}

El crecimiento desigual de los distintos tipos de actividades modifica constantemente los perfiles de especialización económica al tiempo que define las trayectorias seguidas por cada ciudad en el proceso de recomposición del mix de actividades económicas.

En esta sección se estudia la distribución espacial de los ocupados en los distintos grupos de actividades y el crecimiento que han registrado entre 1981 y 2001 en su población ocupada. Se utilizan preferentemente valores absolutos ya que éstos proporcionan una imagen de la concentración espacial de las actividades en las capitales, objetivo principal de esta investigación.

En el mapa de la figura 1 se representa la distribución de los ocupados clasificados en dos grandes divisiones: la industria y servicios que hacen «uso intensivo de investigación, conocimiento y tecnología avanzada» (grupos 1 y 3) por un lado, y las actividades manufactureras y de servicios con «baja intensidad de uso de investigación, conocimiento y tecnología avanzada» (grupos 2 y 4), por otro. 
La representación de los valores totales de las actividades consideradas muestra, ante todo, la gran concentración de población ocupada en 2001 en los municipios de Madrid (1.1171.820 ocupados) y Barcelona (599.017), seguidos a gran distancia por Valencia, Zaragoza, Sevilla, Málaga, Murcia, Bilbao y Alicante. Los restantes municipios capitales se hallan por debajo de los 100.000 ocupados: en este tercer grupo destacan algunas capitales autonómicas como Palma de Mallorca, Valladolid, Oviedo, Vitoria y Santander que superan los 40.000 ocupados. Los datos de afiliados a la Seguridad Social en 2011 presentan una secuencia idéntica a la descrita para 2001. Si se tuviese en cuenta el resto de los asentamientos o se utilizasen otras unidades de análisis, como áreas urbanas funcionales, es probable que grado de concentración a escala nacional fuese aún más elevado, aunque variase el orden de las ciudades.

Una característica estructural de primer orden, recogida también en la figura 1 , se refiere a la diferente proporción que en cada ciudad tienen los ocupados en actividades que hacen «uso intensivo del conocimiento científico y de la tecnología avanzada» (grupos 1 y 3 ) de la que representan los ocupados en actividades con «baja intensidad en el uso del conocimiento científico y tecnologías avanzadas» (grupos 2 y 4). En Madrid, Barcelona y Pamplona los ocupados se reparten prácticamente al 50\% en los dos ámbitos de actividad. En las restantes capitales es mayor, aunque con porcentajes variables, el peso de las actividades del segundo subgrupo, lo que indica que estas ciudades tienen una función importante como centros de comercio y de servicios, entre ellos los administrativos; los ocupados en actividades relacionadas con el turismo también engrosan este último grupo, y su peso es significativo en algunas capitales como las insulares.

La distribución descrita se ha originado por el crecimiento diferencial de la población ocupada en los distintos grupos de actividad entre 1981 y 2001 (Figura 2). Un rasgo destacable de esta evolución es el incremento del número de ocupados en actividades «con uso intensivo del conocimiento y de tecnología avanzada» (grupos 1 y 3 ) en todas las capitales. El aumento ha sido considerable en valores absolutos en Madrid (221.633 ocupados), Barcelona (72.515), Zaragoza (55.485), Valencia (48.599) y Sevilla (43.527). Este grupo ha registrado también crecimientos importantes en Málaga, Murcia y Palma de Mallorca. Por otro lado, las actividades con «baja intensidad de uso de conocimiento científico y tecnología avanzada» (grupos 2 y 4) presentan, en general, cifras de crecimiento inferiores a las del grupo anterior. Si bien Madrid, Zaragoza, Murcia y Sevilla registran aumentos de cierta entidad, los crecimientos son muy modestos en otras capitales, entre las que figura Barcelona. 


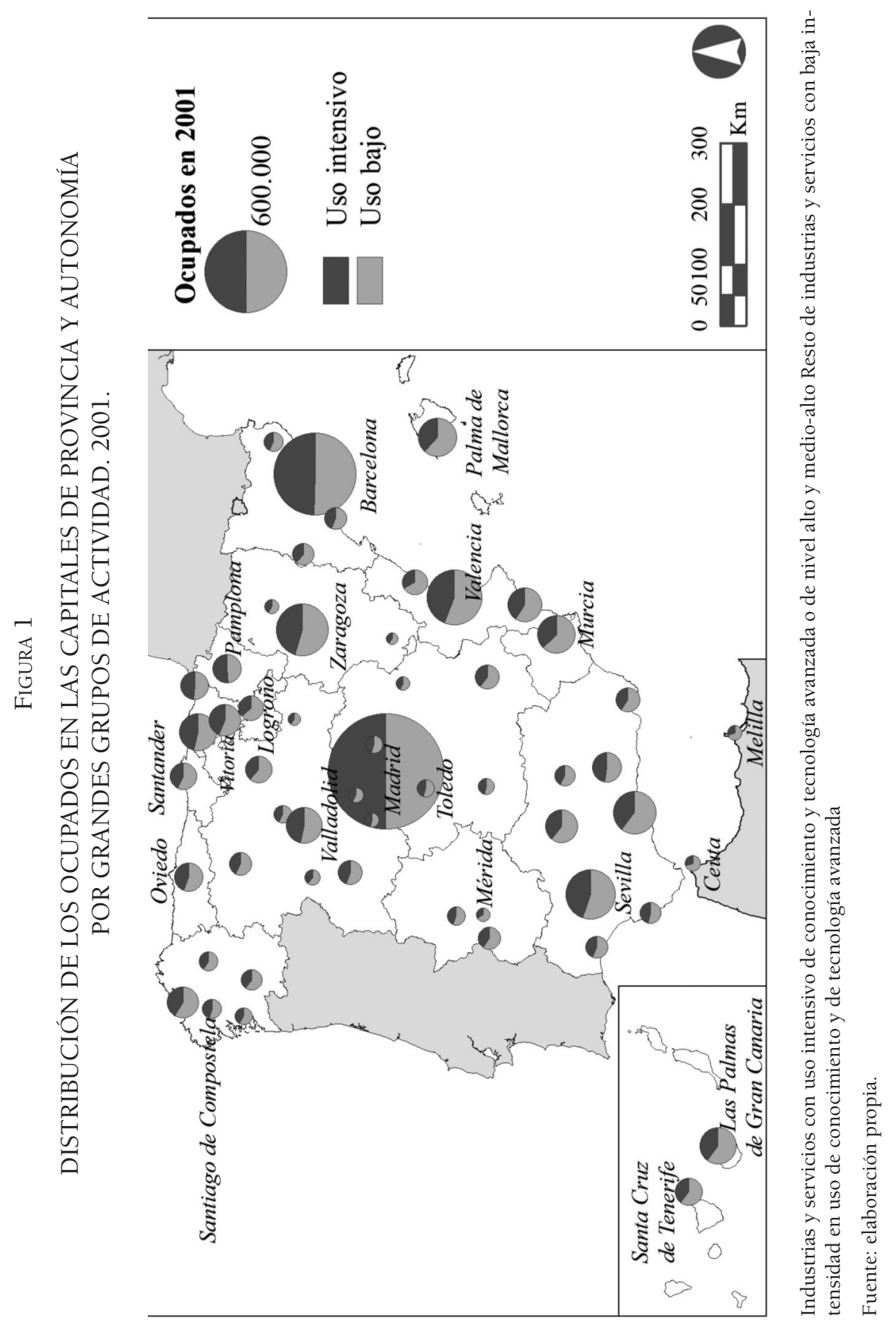

Estudios Geográficos, Vol. LXXV, 276, pp. 139-175, enero-junio 2014 ISSN: 0014-1496, eISSN: 1988-8546, doi: 10.3989/estgeogr.201404 


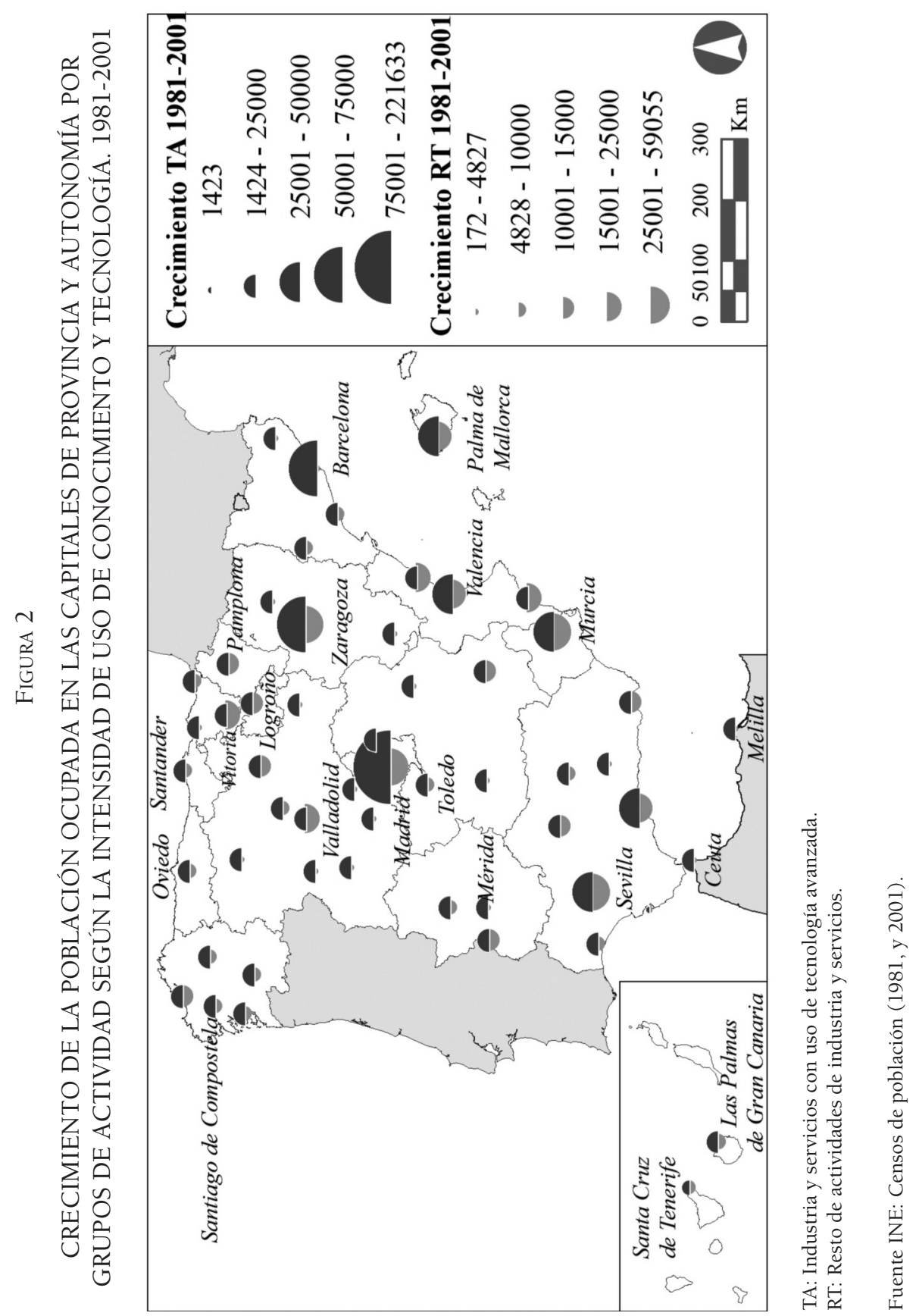

Estudios Geográficos, Vol. LXXV, 276, pp. 139-175, enero-junio 2014

ISSN: 0014-1496, eISSN: 1988-8546, doi: 10.3989/estgeogr.201404 
Esta dualidad de trayectorias por grandes grupos de actividades confirma, por una parte, que los espacios centrales de las grandes aglomeraciones urbanas reúnen ventajas de localización para empresas relacionadas con la creación, utilización y difusión del conocimiento y las innovaciones tecnológicas y, por otra, la «suburbanización» del comercio y servicios, entre ellos los servicios colectivos para la población, siguiendo la dispersión periférica de la población de las grandes ciudades.

Los servicios orientados al mercado y los de salud y educación siguen también dos modelos contrapuestos: el crecimiento de los servicios orientados al mercado (básicamente servicios para las empresas) en todas las ciudades y el decrecimiento o crecimiento modesto de los servicios sanitarios y educativos. Esta evolución es acorde con el proceso de salida de la población de las áreas centrales de las ciudades y la intensificación en estas mismas áreas de actividades de servicios; en algunos casos, el leve crecimiento de la población de las áreas centrales de las grandes ciudades (López, 2011) puede justificar también los ligeros crecimientos de los ocupados en los servicios colectivos par la población.

Todavía es más significativa la oposición entre las actividades industriales con «investigación intensiva y uso de tecnología avanzada» (grupos 1.1. y 1.2.) y los servicios con uso de "conocimiento intensivo y tecnología avanzada» (grupos 3.1. y 3.2.) representadas en el mapa de la figura 3 (compárese con el de la figura 1). Este tipo de servicios crece en todas las capitales y en especial en las más grandes. La industria sólo asciende en algunas capitales y decrece en la mayoría. La combinación de ambas tendencias da lugar a la convergencia en el crecimiento de industria y servicios con uso intensivo de investigación, conocimiento y tecnologías avanzadas en algunos municipios capitales, entre los que destacan los del eje del Ebro (Vitoria, Pamplona, Logroño, Zaragoza), Palma de Mallorca y Murcia, y a la divergencia entre ambos grupos de actividades, por decrecimiento de la industria, en los demás municipios, incluidos Madrid, Barcelona, Valencia y Sevilla.

El análisis por grupos de actividades permite identificar las ciudades con mayor potencial de desarrollo económico-social en función de la estructura y tipos de actividad que en ellas se localizan. En el mapa de la figura 4, que representa la distribución de actividades industriales y de servicios «con uso intensivo de investigación, conocimiento y tecnología avanzada», se refleja de nuevo la polarización ya observada entre Madrid y Barcelona, por un lado, y el resto de las capitales por otro; entre éstas, Zaragoza Valencia y Sevilla constituyen los centros más importantes. En las ciudades mencionadas, pero en especial en Madrid y Barcelona, destaca el peso del grupo 3.2. que contiene los servicios avanzados a las empresas. 


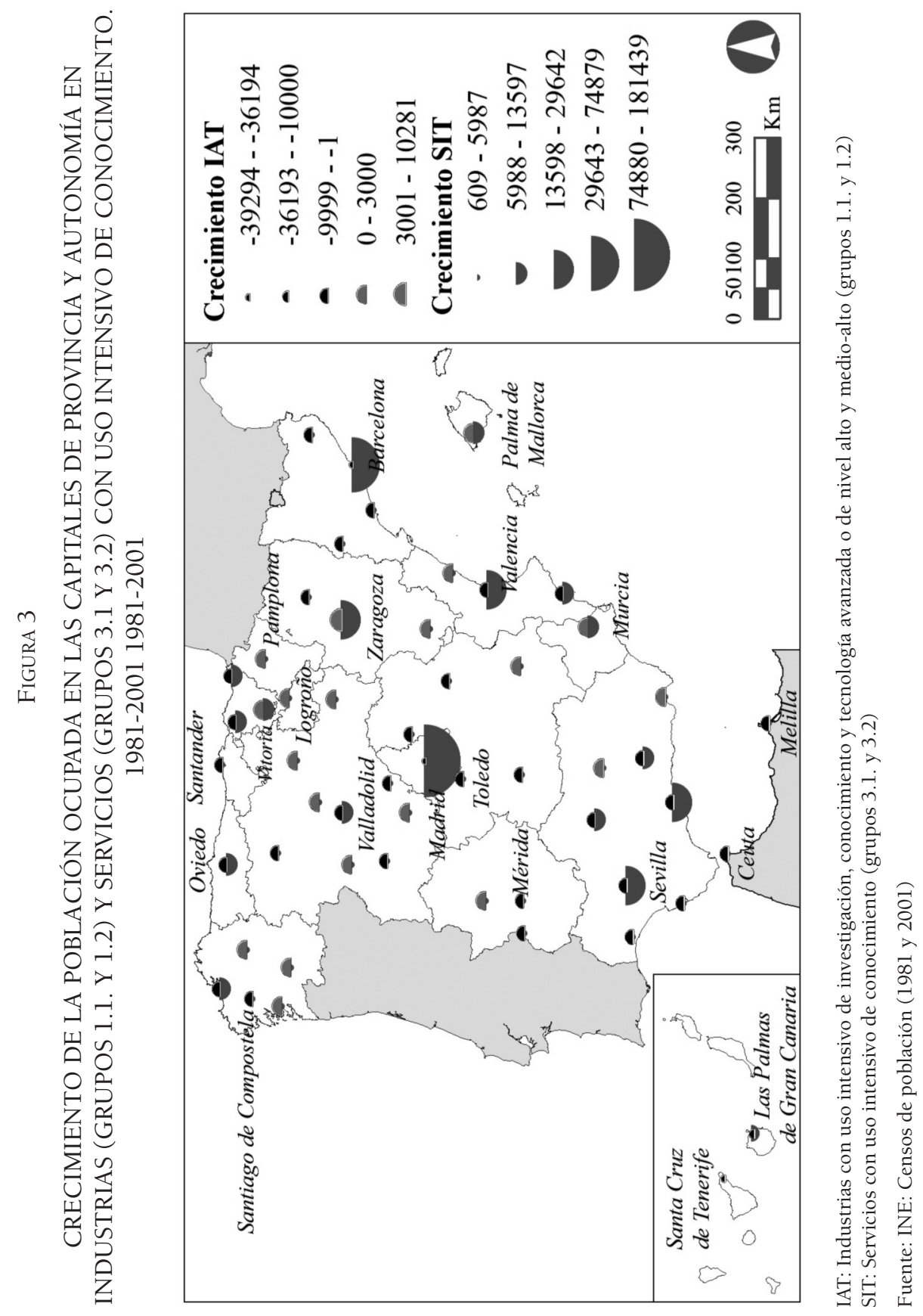

Estudios Geográficos, Vol. LXXV, 276, pp. 139-175, enero-junio 2014

ISSN: 0014-1496, eISSN: 1988-8546, doi: 10.3989/estgeogr.201404 


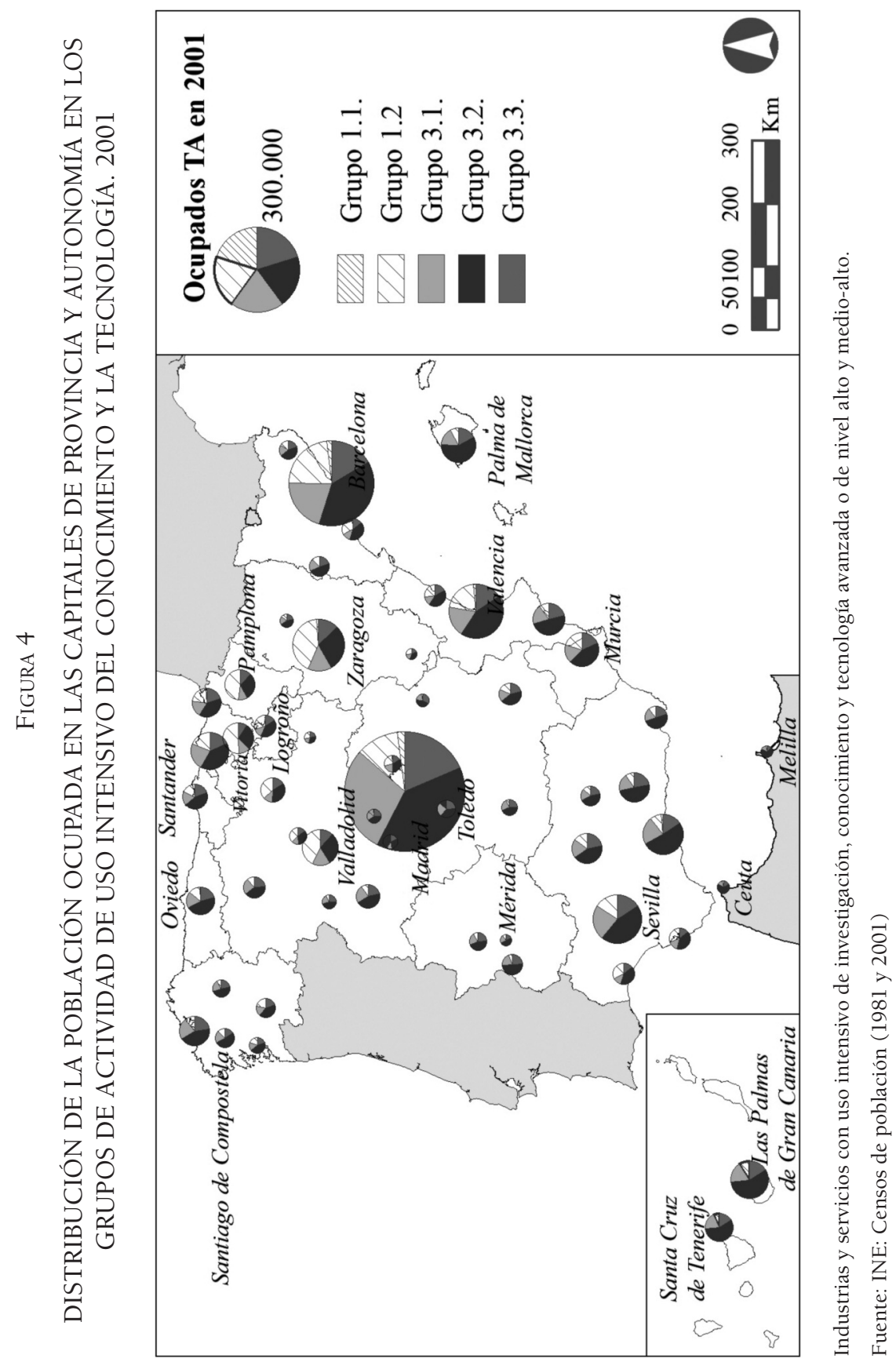

Estudios Geográficos, Vol. LXXV, 276, pp. 139-175, enero-junio 2014 ISSN: 0014-1496, eISSN: 1988-8546, doi: 10.3989/estgeogr.201404 
La composición de los perfiles de especialización económica de las capitales provinciales y de autonomía ha registrado cambios significativos en las tres últimas décadas que pueden resumirse por dos vectores interrelacionados, uno funcional y otro espacial. Por una parte, las actividades de servicio con uso intensivo de conocimiento y tecnología avanzada se concentran de forma muy selectiva en unas cuantas ciudades; la industria intensiva en investigación y en tecnología avanzada o de nivel medio-alto presenta también patrones espaciales bien definidos. Por otra, en el resto de las capitales se mantienen la inercia del peso del comercio y los servicios tradicionales a los que se han añadido servicios colectivos a la población, de salud, educación, de asistencia social, o culturales.

Los resultados de los análisis llevados a cabo se alinean con los obtenidos por Méndez, Sánchez et al. (2009: 128) y prueban que las áreas centrales de las grandes ciudades (en este caso definidas por los límites del municipio capital) son espacios que reúnen ventajas para la localización de actividades de servicios relacionadas con la creación y difusión del conocimiento, la innovación tecnológica y la creatividad. También concuerdan con las conclusiones del estudio de Cuadrado y Maroto (2012), aunque este trabajo utiliza las comunidades autónomas -y no las ciudades- como unidades de análisis.

Es difícil separar el grado y tipo de influencia de cada uno de los factores que hacen más o menos atractivos estos espacios centrales, o municipios, para la localización de las empresas. Sin embargo, la magnitud y diversidad de los mercados de oferta y demanda, el tamaño demográfico y la buena conectividad asegurada por los transportes y comunicaciones, son componentes básicos que explican la predilección de las empresas por estas áreas. En este sentido, es probable que la consolidación de la red Alta Velocidad Ferroviaria (AVE) promueva reajustes en la distribución interurbana de actividades económicas en Zaragoza, Valladolid, Toledo y Guadalajara (Ureña, Coronado et al., 2012), especialmente en los servicios avanzados para las empresas. Asimismo, los efectos de atracción ejercidos por la capitalidad de autonomía son más significativos en las ciudades de dimensión demográfica intermedia.

\subsection{Localización de actividades y tamaño demográfico de los municipios}

Las innovaciones tecnológicas y de organización aplicadas a los sistemas de producción y distribución y a los modos de consumo han variado los requisitos de localización de las actividades económicas, tanto a escala interurbana como intraurbana. 
Los movimientos de centralización y descentralización de actividades habidos entre ciudades guardan relación con el tamaño de las éstas y con su posición en las redes y jerarquías urbanas. En este apartado se examinan las variaciones de la relación entre el tamaño demográfico de las capitales y el tipo y magnitud de las actividades que en ellas se localizan. La población total (x) se utiliza como estimador de la dimensión de cada ciudad y la población ocupada (en 1981 y 2001) y los afiliados a la Seguridad Social (en 2011) como indicadores de la magnitud de cada grupo de actividad (y). Las variables se han ajustado mediante una función potencial.

Los resultados muestran tendencias a la concentración en grandes municipios capitales y a la dispersión por otros más pequeños, lo que es congruente con los crecimientos de las actividades analizadas antes.

En las industrias «con intensidad baja en investigación y tecnología de nivel medio bajo» (grupo 2.1.), el exponente decrece de forma leve y continuada hasta alcanzar en 2011 un valor inferior a la unidad, lo que indica que la presencia de estas actividades en un municipio se reduce proporcionalmente con el incremento de la población. Una tendencia similar puede observarse en la industria con «uso intensivo de investigación y tecnología de nivel medio-alto» (grupo 1.2). La evolución también ha sido similar en las actividades de «servicios con intensidad baja en uso del conocimiento» (grupo 4.1). Los servicios sanitarios, educativos y culturales (grupo 3.4.) mantienen un exponente casi constante entre 1981 y 2011, aunque por debajo de la unidad. En general, los valores del exponente inferiores a 1 expresan relaciones infra-lineales entre el tamaño demográfico y la intensidad de las actividades. En el caso de los servicios sanitarios, educativos y culturales la ligera variación temporal muestra la persistencia de dotaciones de estos servicios proporcionalmente más elevadas en las ciudades pequeñas que en las mayores, aunque con tendencia a la homogeneización.

Los servicios financieros (grupo 3.3.) registran movimientos moderados hacia la concentración en los municipios más grandes.

Los servicios con «uso intensivo de conocimiento orientados al mercado y las empresas» (grupo 3.2.) muestran una marcha firme hacia la concentración creciente en los municipios de mayor tamaño demográfico. En todos los años considerados, los valores del exponente de la ecuación de ajuste se sitúan por encima de la unidad, lo que confirma que los municipios más grandes son los principales centros de oferta de los servicios para las empresas y de los servicios relacionados con la investigación y la innovación tecnológica.

La localización de la industria con «uso intensivo de investigación y tecnología avanzada» (grupo 1.1.) evidencia pautas similares a la de los servicios de 
los dos grupos anteriores, aunque la concentración en grandes municipios es aún más acusada. La reducción del valor del exponente entre 2001 y 2011 indica cierto grado de descentralización de este tipo de industrias desde los municipios más grandes a otros intermedios

Los valores del exponente de la función que ajusta la relación entre los ocupados o afiliados a la Seguridad Social y la población de los municipios capitales de autonomía y de provincia, pueden ser clasificados en dos grandes grupos. Uno incluye los servicios colectivos para la población (sanitarios, educativos, culturales), los servicios «con intensidad baja en uso del conocimiento» (grupo 4.1.) y las «industrias con intensidad baja en investigación y tecnología de nivel medio-bajo» (grupo 2.1.), cuya presencia en cada capital guarda relación directa con su población e, incluso, la proporción de estas actividades es mayor en los municipios más pequeños que la correspondiente al peso de su población; además, en estas actividades apenas han variado la forma de esta relación entre 1981 y 2011. El otro, comprende las restantes actividades industriales y de servicio que hacen uso intensivo de la investigación, el conocimiento y la tecnología avanzada o de nivel medio-alto (grupos 1.1., 1.2. y 3.1.) y las actividades financieras (grupo 3.2.), cuyos valores del exponente indican claras tendencias a la concentración en los municipios más grandes.

La relación estudiada demuestra que las actividades tanto las industriales como de servicios con uso intensivo de investigación, conocimiento y tecnología avanzada se concentran progresivamente en los municipios grandes, donde la accesibilidad a personas y otros servicios es considerable. También evidencia la estabilidad de los servicios colectivos a la población y sus dotaciones proporcionalmente más elevadas en los municipios más pequeños.

\subsection{Trayectorias de especialización: convergencia y diferenciación de los perfiles de actividad}

El crecimiento diferencial de los grupos de actividad en cada capital ha producido una estructura particular en cada una de ellas. En este parágrafo pretendemos construir los perfiles de especialización de cada ciudad según grupos de actividad y resumirlos en grandes categorías para delimitar las trayectorias seguidas entre 1981 y 2011. Este análisis permite identificar los grupos de actividad que tienden a asociarse en cada localización, revelar posibles patrones espaciales y funcionales en las capitales así como distinguir los modelos de cambio de especialización seguidos por cada una de ellas. 
Los perfiles de especialización se han obtenido a partir del coeficiente de localización de cada grupo de actividad calculado en relación con el conjunto de las capitales, por lo que la estructura y valores de especialización de cada capital reflejan su desviación relativa respecto de los valores promedio de todas capitales, que es el objetivo buscado, pero no del total general de los asentamientos de España.

En términos generales se puede afirmar que entre 1981 y 2011 las actividades industriales y las de servicios han seguido patrones diferentes en cuanto a su especialización y localización en los municipios capitales de autonomía y de provincia. Por un lado, en la industria ha disminuido el grado de especialización en varios municipios capitales hasta el punto de desaparecer como actividad especializada, y se ha concentrado en otras, especialmente del centro y norte de España y del eje del Ebro. Por otra, los servicios muestran una pauta dual: la especialización en servicios con uso intensivo de tecnología avanzada y los servicios a las empresas aparece de forma muy selectiva, mientras que los servicios colectivos para la población, el comercio, la hostelería, la administración pública, los servicios personales y otros rebajan su intensidad de especialización, pero está raramente desaparece de las capitales (figuras 5 y 6 ).

Estos cambios en la especialización económica de las capitales obedecen, entre otros procesos, a la reconversión industrial, a la salida de industrias de las áreas centrales de las ciudades, a factores relacionados con políticas industriales y territoriales a diversas escalas, así como a la mejora de las dotaciones de servicios públicos educativos, sanitarios, sociales y culturales, que han reducido las desigualdades sociales y territoriales.

En 2011 (y también en 2001) en la estructura de los perfiles de especialización sobresalen los valores de las actividades industriales. Algunas capitales están especializadas en un grupo de actividad: así Málaga, San Sebastián, Toledo, Valencia, Gerona y Cuenca lo están en actividades con «uso intensivo de investigación y de tecnología avanzada» (grupo 1.1.); Pamplona, Barcelona y Ávila se especializan en actividades con «uso intensivo de investigación y nivel de tecnología medio-alto» (grupo 1.2.); finalmente, Córdoba, Albacete, Murcia, Palencia, Lugo, Orense y Castellón lo hacen en industrias con «intensidad en investigación media-baja y baja» (grupo 2.1.). En otras son dos o más grupos de actividad industrial los que tienen algún grado de especialización como en Tarragona, Zaragoza, Logroño, Vitoria, Burgos, Valladolid y Sevilla. En 2011 ha desaparecido la especialización industrial en Santander, Cádiz y Málaga respecto de 2001 y 1981.

Un rasgo notable de los perfiles de especialización de 1981 (figura 5) se encuentra en la destacada presencia de los servicios con uso intensivo del cono- 
cimiento y de tecnología avanzada (grupo 3.1.) que en este año está compuesto exclusivamente por la rama de actividad: 76. "Comunicaciones». Como ya se advirtió, los avances tecnológicos han hecho cambiar, radicalmente, la organización y las prácticas en estas actividades, parte de las cuales están integradas de forma estratégica en las propias empresas (por ejemplo en la banca, finanzas y seguros).

Otra característica destacable para el conjunto de los servicios es el descenso generalizado de los valores del coeficiente de localización habido entre 1981 y 2011; esta tendencia traduce la paulatina equiparación de las dotaciones de estos servicios entre las capitales, especialmente en los servicios colectivos para la población y en otras actividades comerciales, de transporte y servicios con baja intensidad en uso del conocimiento. La especialización en servicios persiste en las capitales extremeñas, de Castilla-La Mancha, de Castilla y León, de Oviedo, Santander y otras de Andalucía. En efecto, en los mapas de las figuras 5 y 6 se recoge la reducción de los valores de especialización de estos grupos de servicios junto con la persistencia de los servicios colectivos a la población como grupo especializado, aunque con valores bajos del coeficiente de localización.

Una tercera cualidad esencial de la especialización en servicios reside en la extraordinaria concentración de los «servicios» con «uso intensivo de conocimiento y tecnología avanzada» (grupo 3.1.) y «servicios con uso intensivo de conocimiento orientados al mercado y a las empresas» (grupo 3.2.) que en 2011 sólo aparecen como actividades especializadas en los perfiles de Madrid, Barcelona, A Coruña, Santiago y Gerona. En 2001 estos grupos de servicios presentaban algún grado de especialización en Madrid, Barcelona, Bilbao y Palma de Mallorca.

Los servicios financieros tienen bajos índices de especialización aunque aparecen en trece capitales, es decir, ocupan una posición intermedia entre las dos grandes agrupaciones de servicios antes mencionados.

La variabilidad de las estructuras de especialización se puede generalizar en cuatro grandes tipos según el predominio de las actividades industriales, de servicios o de ambas, o bien según perfiles en los que no domine un tipo particular de actividad. Los perfiles específicos de especialización y su tipificación son indicadores importantes para conocer la fase de incorporación de cada capital a la economía del conocimiento en el contexto del conjunto de las capitales españolas, así como para trazar las trayectorias de especialización seguidas por cada una. Tanto la determinación de la estructura como de la trayectoria de especialización son datos relevantes para diseñar acciones y políticas estratégicas que conformen la orientación futura de la actividad eco- 


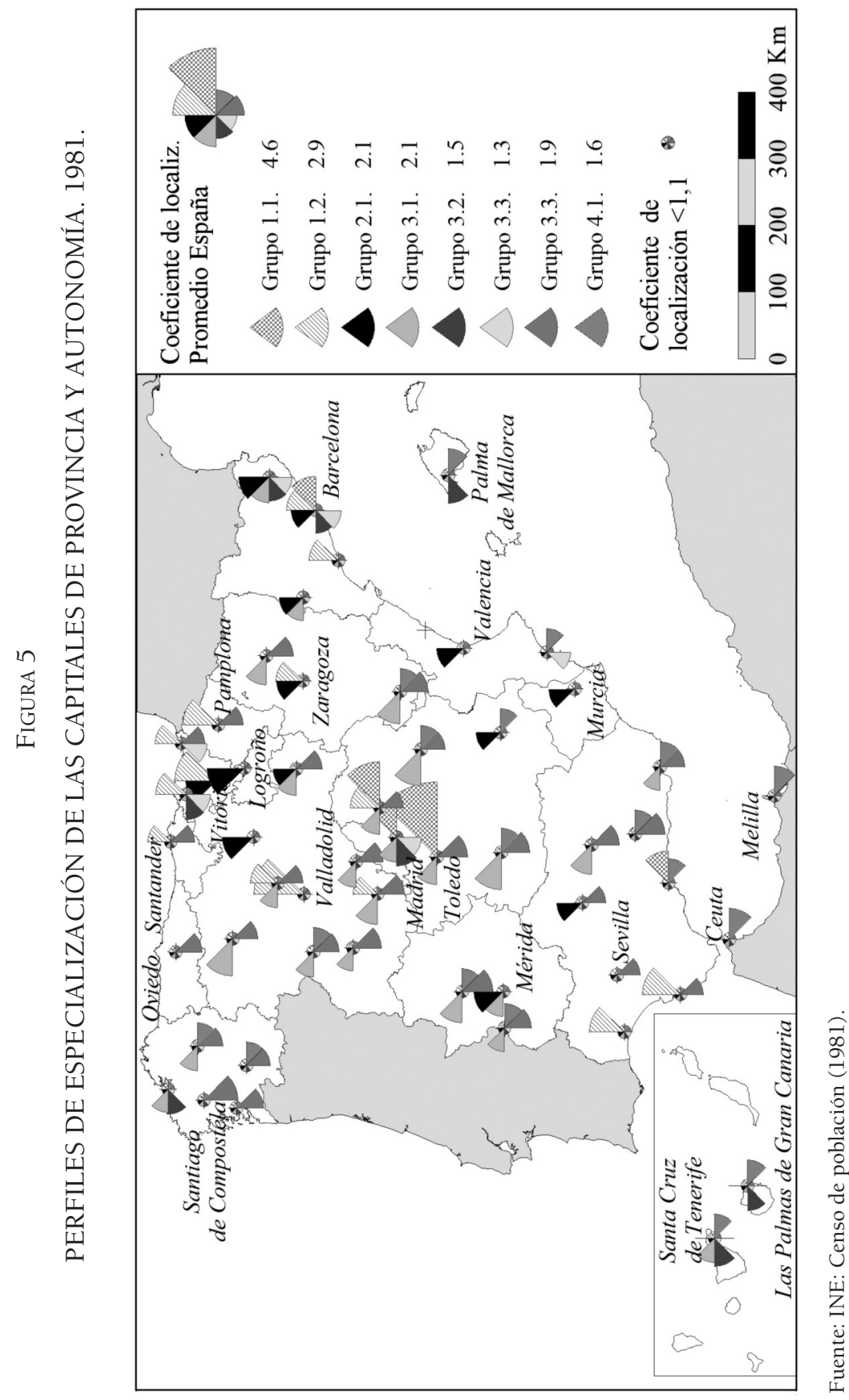

Estudios Geográficos, Vol. LXXV, 276, pp. 139-175, enero-junio 2014 ISSN: 0014-1496, eISSN: 1988-8546, doi: 10.3989/estgeogr.201404 


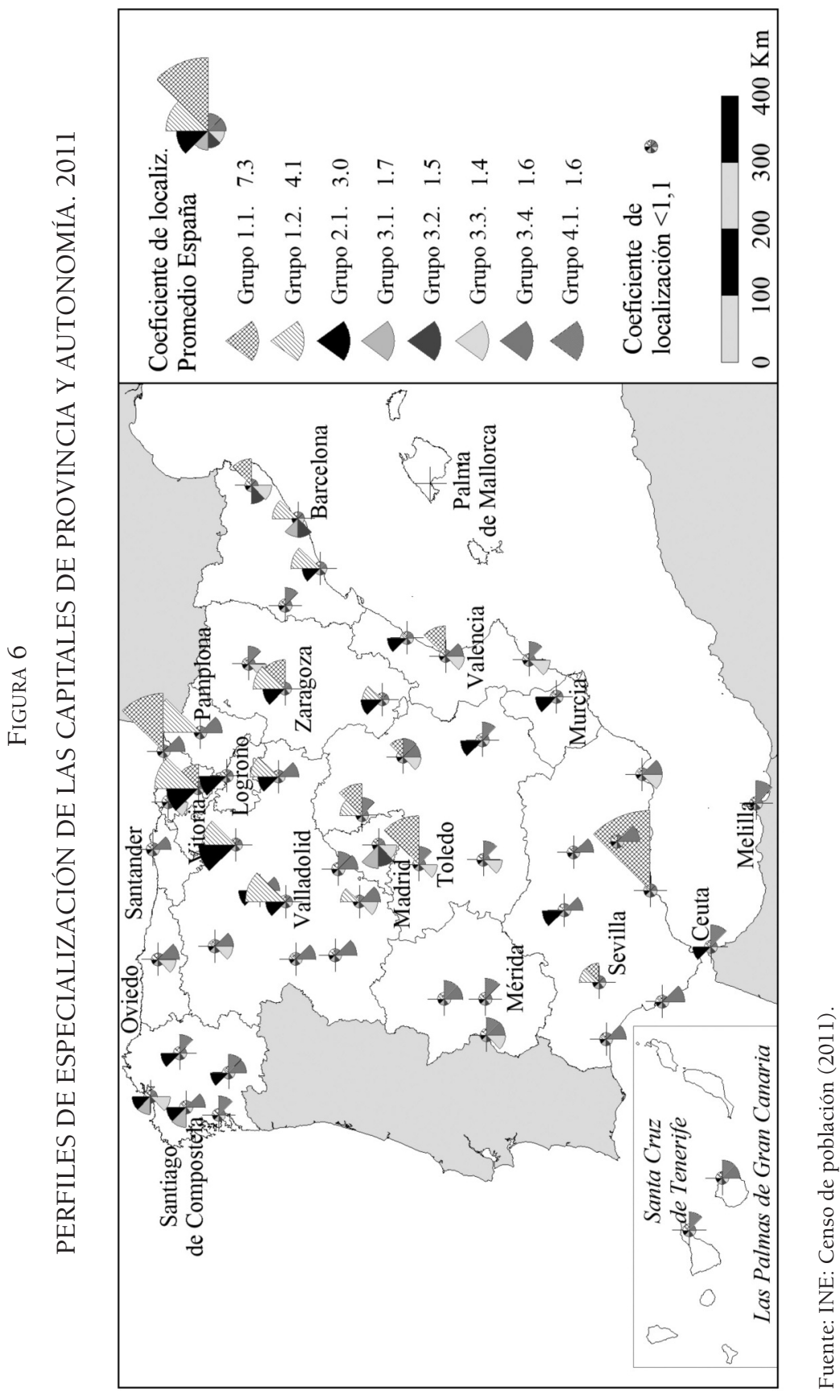

Estudios Geográficos, Vol. LXXV, 276, pp. 139-175, enero-junio 2014

ISSN: 0014-1496, eISSN: 1988-8546, doi: 10.3989/estgeogr.201404 
nómica en relación con la investigación, el conocimiento y la innovación como fuerzas impulsoras del desarrollo socioeconómico.

En 2011, las capitales en las que predominan las actividades industriales se localizan en el centro y norte de España y en el eje del Ebro, salvo Sevilla y Málaga. Esta distribución es muy similar a la de 2001, de la que han desaparecido Cádiz y Huelva que si figuraban en 1981 como municipios con especialización industrial; en 2001 Toledo no poseía especialización en industria como si la tiene en 2011.

El dominio de los servicios en la estructura de especialización es lo más frecuente entre las capitales, aunque existen diferencias en los grupos de servicios predominantes: así en Madrid destacan los «servicios con uso intensivo del conocimiento y tecnología avanzada» (grupo 3.1.) que también están presentes, en menor grado, en A Coruña y Santiago, frente a las restantes capitales en cuyos perfiles destacan los servicios colectivos a la población (grupo 3.4.) y los «servicios con intensidad baja en el uso del conocimiento» (grupo 4.1.).

Otro modelo de estructura de perfiles de especialización es el definido por la participación conjunta de actividades industriales y de servicios. Se corresponden con este modelo las economías diversificadas de las grandes capitales como Barcelona, Valencia, Sevilla y Bilbao y de otras ciudades intermedias y pequeñas como Pamplona, San Sebastián, Tarragona, Girona, Toledo y Soria.

En las restantes capitales no aparece ninguna actividad dominante en su perfil de especialización.

La composición de los perfiles de especialización de cada capital en 2011 representa un estado resultado de las variaciones de cada grupo de actividad medidas en relación con el conjunto de las capitales. Los perfiles de partida son los de 1981 y los finales los de 2011; un punto de comparación intermedio se sitúa en 2001.

La evolución de la estructura de especialización de las capitales ha seguido alguna de las cuatro trayectorias representadas en los datos de la tabla 4.

A. Estable (15 capitales). Este recorrido se caracteriza por la permanencia en el tiempo del tipo de especialización; los grupos más numerosos son de especialización en servicios y en industria y servicios. En esta clase se incluyen la mayor parte de las grandes capitales (excepto Madrid y Sevilla) y más de 2/3 de las capitales autonómicas.

B. Estable y variación reciente ( 7 capitales). Esta categoría se define por la continuidad de la especialización entre 1981 y 2001 y el cambio entre este año y 2011. En general, se trata de casos específicos en los que la reorientación de la especialización económica se debe al estancamiento o retroceso de algunas actividades (por ejemplo la industria en Huelva y Madrid) o el avance de otras (por ejemplo los servicios en Pamplona y Girona). 
TABLA 4

TRAYECTORIA DE ESPECIALIZACIÓN ECONÓMICA DE LAS CAPITALES DE PROVINCIA Y AUTONOMÍA ENTRE 1981 Y 2011

\begin{tabular}{|c|c|c|c|}
\hline $\begin{array}{c}\text { Trayectoria } \\
\text { en el año: } \\
\text { 1981-2001-2011 }\end{array}$ & Capitales & $\begin{array}{c}\text { Trayectoria } \\
\text { en el año: } \\
\text { 1981-2001-2011 }\end{array}$ & Capitales \\
\hline \multicolumn{2}{|l|}{ A. Estable } & \multicolumn{2}{|c|}{ C. Variable y estabilidad reciente } \\
\hline $0-0-0$ & Ceuta, Melilla & $2-0-0$ & $\begin{array}{l}\text { Albacete, Córdoba, } \\
\text { Cuenca, Lugo, Lleida, } \\
\text { Murcia }\end{array}$ \\
\hline $1-1-1$ & Valladolid, Vitoria & $3-1-1$ & Burgos, Zaragoza \\
\hline $2-2-2$ & $\begin{array}{l}\text { Alicante, A Coruña, } \\
\text { León, Oviedo, Palma de } \\
\text { Mallorca, Las Palmas de } \\
\text { Gran Canaria, Santa } \\
\text { Cruz de Tenerife }\end{array}$ & $3-2-2$ & Santander \\
\hline 3-3-3 & $\begin{array}{l}\text { Bilbao, Barcelona, } \\
\text { San Sebastián, Valencia }\end{array}$ & & \\
\hline \multicolumn{2}{|c|}{ B. Estable y variación reciente } & \multicolumn{2}{|l|}{ D. Fluctuante } \\
\hline $1-1-0$ & Huelva & $1-3-2$ & Cádiz \\
\hline $1-1-3$ & Pamplona & $2-1-0$ & Logroño \\
\hline $2-2-0$ & Segovia & $2-0-2$ & $\begin{array}{l}\text { Almería, Badajoz, } \\
\text { Cáceres, Ciudad Real, } \\
\text { Granada, Huesca, Jaén, } \\
\text { Mérida, Ourense, } \\
\text { Pontevedra, Salamanca, } \\
\text { Santiago, Zamora }\end{array}$ \\
\hline $2-2-3$ & Gerona & $2-3-0$ & Ávila, Teruel \\
\hline 3-3-1 & Guadalajara, Málaga & $2-3-1$ & Soria \\
\hline \multirow[t]{3}{*}{$3-3-2$} & Madrid & $3-1-0$ & Palencia, Castellón \\
\hline & & $3-1-3$ & Tarragona \\
\hline & & $3-2-3$ & Sevilla, Toledo \\
\hline
\end{tabular}

0: Sin especialización definida; 1: Predominio de actividades industriales; 2: Predominio de actividades de servicios; 3: combinación de industria y servicios; (negrita y cursiva: capitales de autonomía y ciudades autónomas)

Fuentes: INE (1981 y 2001): Censos de población. Seguridad Social (2011): Afiliados a la Seguridad Social (todos los regímenes). Elaboración propia. 
C. Variable y estabilidad reciente (9 capitales). La marcha de la especialización de este grupo de ciudades se distingue por el cambio producido entre 1981 y 2001 y la posterior permanencia hasta 2011. Se incluye en esta agrupación capitales de tamaño demográfico y económico medio y pequeño, de economías no especializadas (Lugo, Cuenca, Lérida, Murcia...) junto a otras de economía bastante especializada en industria (Zaragoza) o servicios (Santander).

D. Fluctuante (23 capitales). Las capitales de este grupo han trazado un vector cuya dirección de especialización varía en cada año considerado. Este itinerario se explica por el crecimiento desigual en cada período de los grupos de actividad; así, en 2011 hay un retorno a la especialización de 1981 en varias capitales, entre ellas en Mérida y Santiago, o bien se varía la especialización por retroceso de la industria (Cádiz); en los restantes casos, el cambio de especialización se debe al incremento de los servicios.

La evolución de la estructura de los perfiles de especialización de actividad económica entre 1982 y 2011, reflejada en los mapas de las figuras 5 y 6 y en la tabla 4, está compuesta por trayectorias heterogéneas, correspondientes a las ciudades que han variado significativamente sus perfiles de especialización frente a otras caracterizadas por su estabilidad.

Las variaciones más importantes de las estructuras de especialización se deben a la presencia (e intensidad mayor o menor) de «actividades industriales intensivas en investigación y con uso de tecnología avanzada», a los «servicios con uso intensivo de conocimiento y tecnología avanzada y a los servicios con uso intensivo de conocimiento y tecnología avanzada orientados al mercado y a las empresas». En cambio, los «servicios a la población» y los «servicios con baja intensidad en uso del conocimiento» registran una considerable inercia temporal y espacial.

La combinación de las tendencias señaladas ha producido grupos de perfiles de especialización asociados a sus correspondientes patrones espaciales. Uno de ellos se caracteriza por el predominio de los servicios colectivos a la población y por otros servicios con baja intensidad en uso del conocimiento (comercio, hostelería, reparaciones en general); este grupo es el más estable tanto en el tiempo como en el espacio. Otro grupo se identifica por la presencia de actividades industriales intensivas en investigación y con uso de tecnología avanzada o de nivel medio-alto; destaca, en general, por valores elevados de especialización y su concentración espacial en las capitales del centro, norte y noreste de España. El tercero se define por la presencia de servicios con uso intensivo de conocimiento y tecnología avanzada y los servicios con uso intensivo de conocimiento y tecnología avanzada orientados al mercado y a las empresas, muy selectivo en su localización. 
En resumen, los cambios en los perfiles de especialización económica de las capitales de provincia y autonomía habidos en el periodo 1980-2011 han incrementado, en cierto grado, la polarización de las estructuras de especialización entre las capitales que mantienen su especialización basada en servicios a la población y otros de corte tradicional, por un lado, y las que han incorporado actividades industriales y de servicios con uso intensivo de conocimiento y tecnología avanzada, por otro.

La evolución de los perfiles de especialización económica entre 1981 y 2011 no muestra desviaciones significativas asociadas a la capitalidad de autonomía. En todo caso, cabe señalar que en 2011 los «servicios con uso intensivo de conocimiento o y tecnología avanzada» (grupo 3.1.) se han incorporado como actividades especializadas a la estructura económica de Santiago de Compostela.

\section{CONSIDERACIONES FinAleS}

Las estructuras de especialización económica de los municipios capitales de autonomía y provincia se han modificado en los últimos 30 años producto de las variaciones diferenciadas de las distintas actividades económicas.

Los análisis precedentes muestran que la configuración de los perfiles de especialización propios de economías del conocimiento se limita a un número reducido de capitales. La formación de este tipo de estructura se debe, obviamente, al incremento del peso de la industria y los servicios con uso intensivo de investigación, del conocimiento y de tecnología avanzada, especialmente de los servicios orientados al mercado y a las empresas.

La industria con uso intensivo de investigación y tecnología avanzada o de nivel medio-alto muestra cierto grado de concentración, por lo que su presencia marca intensamente la especialización de varias capitales, la mayor parte situadas en el centro-norte de España y en el eje del Ebro.

Más selectiva es aún la localización de los «servicios con uso intensivo de conocimiento y tecnología avanzada» y «los servicios con uso intensivo del conocimiento orientados al mercado y a las empresas» que sólo definen, con claridad, las siluetas de especialización de Madrid y Barcelona.

Los cambios en la estructura de especialización han sido más fuertes en la década 2001-2011 que durante los 20 años que separan 1981 de 2001. Considerando ambos periodos, 2/3 de las capitales ha variado su especialización siguiendo trayectorias diferentes. La especialización más frecuente de las capitales es en actividades de servicio; le siguen en número aquellas capitales 
que no tienen especialización definida. Precisamente, la especialización en servicios es la más estable en el tiempo, que se ha reforzado por el incremento generalizado de los servicios colectivos a la población, lo que ha ido igualando las dotaciones de las capitales (más elevadas, en términos relativos, en las capitales más pequeñas) y atenuando el nivel de especialización de estas actividades. Esta ha sido la tendencia seguida por la mayor parte de las capitales de provincia y autonomía.

En relación con la principal hipótesis de este trabajo, el estudio llevado a cabo permite constatar la tendencia a la concentración en determinadas capitales de las actividades que definen la economía del conocimiento. La buena conectividad de las ciudades o la cercanía a grandes áreas urbanas son, entre otros, factores de atracción de este tipo de actividades; la capitalidad de autonomía tiene una influencia limitada en ciudades de tamaño medio, ubicadas en contextos regionales dinámicos.

Fecha de recepción: 04/11/2013.

Fecha de aceptación: 20/02/2014.

\section{Bibliografía}

Acs, Z. J. (ed.) (2006): The growth of cities. Cheltenham, Edward Elgar.

Bailly, A. S. y Coffey, W. J. (1994): "Localisation des services à la production et resturations économiques. Un analyse théorique". L Espace Géographique, 1, pp. 224-230.

Barcet, A., Bonamy, J. y Mayere, A. (1984): "Les services aux entreprises: problèmes théoriques et méthodologiques". Recherches économiques et sociales. La documentation française, 9, pp. 254-277.

Castells, M. (1995): La ciudad informacional. Tecnologías de la información, reestructuración económica y el proceso urbano regional. Madrid, Alianza Editorial.

Clayton, N. y Morris, K. (2010): Recession, Recovery and Medium-Sized Cities. Londres, 40 p. Disponible en: http://www.theworkfoundation.com/. (Consultada en febrero de 2014).

Coats, D. (2005): "An agenda for work: The Work Foundation's challenge to policy makers". The Work Foundation, 1/2, p. 57. Disponible en: http://www.thework foundation.com/assets/docs/publications/73_Agenda_for_work.pdf. (Consultada en febrero de 2014).

Coats, D. y Lekhi, R. (2008): “Good Work': Job Quality in a Changing Economy". The Work Foundation, 89. Disponible en http://www.theworkfoundation.com/. (Consultada en febrero de 2014). 
Cooke, P.; Asheim, B.; Boschma, R. et al. (eds.) (2011): Handbook of Regional Innovation and Growth. Cheltenham, Edward Elgar Publishing.

Cuadrado, J. y Maroto, A. (2012): "Análisis del proceso de especialización regional en servicios en España”. EURE, 38/114, pp. 5-34.

Drucker, P. F. (1969): The age of discontinuity: guidelines to our changing society. London, Heinemann.

Duranton, G. y Puga, D. (2002): From Sectoral to Functional Urban Specialization. London, Centre for Economic Policy Research (CEPR). Discussion. Paper, 2971, revised version June 2003.

Escolano, S. (2012): "Efectos de la capitalidad autonómica en la dinámica del sistema urbano español", en Valenzuela, M. (coord.), El impacto del modelo autonómico en las ciudades españolas. Una aproximación interdisciplinar. Madrid, Servicio de Publicaciones de la Universidad Autónoma de Madrid, pp. 145-170.

EUROSTAT (2011): Science, technology and innovation in Europe. Luxemburgo, Comisión Europea, EUROSTAT.

EUROSTAT (s/f): Aggregations of manufacturing based on NACE Rev 1.1. EUROSTAT: 2. Disponible en: http://epp.eurostat.ec.europa.eu/cache/ITY_SDDS/Annexes/htec_ esms_an2.pdf (Consultada en febrero de 2014).

Glazer, L. y Grimes, D. (2009): Michigan s Transition to a Knowledge-Based Economy: Second annual Progress Report. Michigan, Future. Disponible en: http://www.michigan future.org/Reports/progress\%20report\%2009.pdf. (Consultada en febrero de 2014).

Halbert, L. (2005): "Les métropoles, moteurs de la dématérialisation du système productif urbain français: une lecture sectorielle et fonctionelle (1982-1999)". Bulletin de 1 Association de Géographes Françaises, 3, pp. 279-297.

Hepworth, M. (1986): "The Geography of Technological Change in the Information Economy". Regionalo Studies, 20/5, pp. 407-424.

INE (s/f): CNAE-93 Rev 1: Clasificación nacional de Actividdes Económicas. 1993 Rev.1. INE: 32. Disponible en: http://www.ine.es/clasifi/cnae93revl.pdf (Consultada en febrero de 2014).

Instituto Geográfico Nacional (2008): Atlas Nacional de España: Demografía. Madrid, Centro Nacional de Información Geográfica.

Jonhston, R. J.; Taylor, P. J. y Watts, M., (eds.) (2002): Geographies of global change: remapping the world (2nd). Oxford, Blackwell.

Korres, G. M. (2009): Technical change and economic growth: Inside the knowledge based Economy 2008. London, Averbury Press.

Korres, G. M.; Tsobanaglou, G. O. y Kokkinou, A. (2011): "Innovation Geography and Regional Growth in European Union”. SageOPen, 1-10, DOI:10.1177/2158244011413142. Disponible en: http://sgo.sagepub.com/con tent/1/1/2158244011413142 (Consultada en febrero de 2014).

Krätke, S. (2007): "Metropilisation of the european Economic Territory as a consequence of Increasing Specialisation of Urban Agglomerations in the Knowledge Economy". European Planning Studies, 15/1, pp. 1-27. 
López, A. (2011): “¿Vuelve el Centro? Caracterización demográfica de los procesos de reurbanización en las metrópolis españolas", en Pujadas, I.; Bayona, J.; García, A.; et al. (eds.): Población y espacios urbanos. Barcelona, Department de Geografia Human de la UB y Grupo de población de la AGE, pp. 163-180. Disponible en http://www.ub.edu/congreso_poblacion/ (Consultada en febrero de 2014).

Mandeville, T. (1983): "The Spatial Effects of Informational Technology". Futures, 15/1, pp. 65-72.

May, N. (1997): "From territorial production systems to metropolitanization: ten years of research in France", en Moulaert, F. y Scott, A. J. (eds.): Cities, enterprises and society on the eve of 21st century. London, Pinter, pp. 57-77.

Méndez, R.; Michelini, J. J.; Prada, J.; et al. (2012): "Economía creativa y desarrollo urbano en España: una aproximación a sus lógicas espaciales". EURE, 38/113, pp. 5-32.

Méndez, R. y Pascual, H. (eds.) (2006): Industria y ciudad en España: nuevas realidades, nuevos retos. Cizur Menor (Navarra), Aranzadi, Thomsom-Civitas.

Méndez, R.; Sánchez, S.; Abad, L.; et al. (2009): "Sistema urbano y sociedad del conocimiento: hacia una tipología de las ciudades españolas". Investigaciones Regionales, 16, pp. 117-142.

Mitchell, W. J. (1995): City of bits : space, place, and the infobahn. Cambridge, Massachusetts, MIT.

Moreno, A. y Escolano, S. (1992): Los servicios y el territorio. Madrid, Síntesis.

Mos, M. (1998): "Technology and Cities". Cityscape: A Journal of Policy Development and Research, 3/3, pp. 107-127.

Moulaert, F. y Scott, A. J. (1997): "Globalization, economic reestructuring and urban society", en Moulaert, F. y Scott, A. J. (eds.): Cities, enterprises and society on the eve of 21st century. London, Pinter, pp. 3-17.

Puissant, S. y Lacour, C. (2011): "Mid-sized French cities and their niche competitiveness". Cities, 28, pp. 433-443.

Reques, P. (2011): "Transformaciones espaciales y procesos socio-demográficos en la ciudad", en Pujadas, I., Bayona, J., García, A., et al. (eds.): Población y espacios urbanos. Barcelona, Department de Geografia Human de la UB y Grupo de población de la AGE, pp. 235-284. Disponible en: http://www.ub.edu/congreso_poblacion/ (Consultada en febrero de 2014).

Reques, P. y Rodríguez, V. (1998): Atlas de la población española. análisis de base municipal. Santander, Servicio de Publicaciones de la Universidad de Cantabria/CSIC/ ESRI-España.

Scott, A. J. (1997): "The cultural economy of cities". International Journal of Urban and Regional Research, 21/2, pp. 323-339.

Stehr, N. (1994): Knowledge Societies: The Transformation of Labour, Property and Knowledge in Contemporary society. London, Sage.

Storper, M. y Venables, A. J. (2004): "Buzz: face-to-face contact and the urban economy". Journal of The Economic Geography, 4, pp. 351-370.

UNESCO (2005): Towards Knowledge Societies. Paris, UNESCO. 
Ureña, J. M. D. (ed.) (2012): Teritorial Implications of High Speed Rail. A Spanish Perspective. Farnham (UK), Ashgate.

Ureña, J. M. D.; Coronado, J. M.; Garmendia, M.; et al. (2012): "Terrotorial Implications at national and Regional Scales of High-sèed Rail”, en Ureña, J. M. D. (ed.): Terrotorial Implications of High Speed Rail. A Spanish perspective. Farnham (UK), Asghate, pp. 129-162.

Varga, A. (ed.) (2009): Universities, Knowledge Transfer and Regional Development. Geography, Entrepreneurship and Policy. Cheltenham, Inglaterra, Edward Elgar Publising.

Veltz, P. (1997): "The dinamics of production systems, territories and cities", en Moulaert, F. y Scott, A. J. (eds.): Cities, enterprises and society on the eve of 21 st century. London, Pinter, pp. 78-96.

Yigitcanlar, T. (2009): "Spatial reestructuring of cities in the age of knowledge economy: insights from Australian cities", en 2nd Knowledge Cities Summit. Shenzhen, China, QUT Digital Repository.

\section{RESUMEN}

Parte de la reorganización reciente de las actividades económicas en España se define por la concentración en determinadas áreas urbanas de ciertas actividades económicas, especialmente las relacionadas con la creación, gestión y uso de la innovación y el conocimiento, y por la descentralización selectiva de otras.

La hipótesis principal de este trabajo asume que la capitalidad de autonomía es un factor que ejerce influencia en la estructura de las actividades económicas de una ciudad. En este marco se estudia el alcance de estas tesis referidas a los municipios capitales de provincia y de autonomía entre 1981 y 2011. El estudio permite constatar la tendencia a la concentración en determinadas capitales de las actividades de la «economía del conocimiento». La buena conectividad de las ciudades o la cercanía a grandes áreas urbanas son fuerzas de atracción de este tipo de actividades; la capitalidad de autonomía tiene una influencia limitada en núcleos de tamaño intermedio ubicados en contextos regionales dinámicos.

PAlabras Clave: especialización; concentración; innovación; capitales; España.

\section{AbStract}

Some fundamental trends of the recent reorganization of economic activities in Spain are defined by the growing concentration in certain urban areas of certain economic activities, especially those related to the creation, management and use of innovation and knowledge, and selective decentralization of others.

The main hypothesis of this work assumes that capital of autonomy is a factor that exerts some influence on the structure of economic activities in a city. In this paper we study the scope of this thesis referred to municipalities and provincial capitals of autonomy between 1981 and 2011. The study conducted helps verify the trend to- 
wards concentration of capital in certain activities of the knowledge economy. The good connectivity of cities or the proximity to large urban areas are attractive forces; the capital of autonomy has a limited influence on intermediate sized nuclei located in dynamic regional contexts.

KEY WORDS: specialization; concentration; innovation; capital; Spain.

\section{RÉSUMÉ}

La réorganisation récente des activités économiques en Espagne est définie d'un côté par la concentration de certaines d'entre elles, en particulier celles liées à la création, gestion et utilisation de l'innovation et de la connaissance dans des zones urbaines concrètes, et de l'autre, par la décentralisation sélective d'autres activités.

Lhypothèse principale de cette recherche accepte que la «capitalité» d'une région autonome a des conséquences sur la structure des activités économiques de cette ville. Nous allons donc vérifier cette thèse référée aux municipalités capitales de province ou de région autonome dans la période 1981-2011. Cette analyse permettra de confirmer la tendance à la concentration des activités de l'économie de la connaissance dans certaines de ces capitales. Une connectivité performante ou la proximité de grands réseaux urbains constituent des pôles d'attraction pour ces activités; la «capitalité» d'une région autonome a une influence limitée dans le cas de villes moyennes situées dans un contexte régional dynamique.

MOTS-CLÈS: spécialisation; concentration; capital; innovation; l'Espagne. 